\title{
Sidereal orientation of the Earth and stability of the VLBI celestial reference frame
}

\author{
M. Feissel-Vernier ${ }^{1,2}$, C. Ma ${ }^{3}$, A.-M. Gontier ${ }^{4}$, and C. Barache ${ }^{4}$ \\ 1 Observatoire de Paris/SYRTE, CNRS UMR 8630, 61 Av. de l'Observatoire, 75014 Paris, France \\ 2 Institut Géographique National/LAREG, 8 Av. Blaise Pascal, Champs-sur-Marne, 77455 Marne-la-Vallée Cedex 2, France \\ e-mail: feissel@ensg.ign.fr \\ 3 NASA/Goddard Space Flight Center, USA \\ e-mail: chopo.ma@nasa.gov \\ 4 Observatoire de Paris/SYRTE, CNRS UMR 8630, France \\ e-mail: [anne-marie.gontier; christophe. barache]@obspm.fr
}

Received 19 October 2004 / Accepted 1 February 2005

\begin{abstract}
The consideration of time stability of extragalactic radio sources observed by VLBI is shown to allow the realisation of more consistent celestial reference frames. The impact on the estimation of precession and nutation components is investigated over the time span 1984-2002. The precession correction to the IAU 2000 value that is obtained when excluding the unstable sources reaches $49 \pm 5 \mu$ as/year, to be compared to $12 \pm 5 \mu$ as/year using the current conventional celestial frame. The determination of the obliquity rate is unaffected and remains at the level of $27 \pm 2 \mu$ as/year. The observed correction to the 18.6-year nutation amplitude using the current conventional celestial frame ie sizeably corrupted by the unstable sources. After accounting for this effect, the estimations relative to both sets of reference radio sources confirm a discrepancy with the IAU 2000 nutation model with a total amplitude of $320 \pm 100 \mu$ as for the observed nutation in longitude, to be compared to the $80 \mu$ as discrepancy found by Mathews et al. (2002, JGRB, 107, 1029). The discrepancy in obliquity amounts to $50 \pm 16 \mu$ as. The effect of source instability is shown to have an impact on the determination of universal time at the one microsecond level. The high and medium frequency nutation terms (up to periods of a few years) are impacted only in the early years of the program.

Chapter 7 concerning the observation of the core and inner core free nutations is paralleled by a twin paper (Dehant et al. 2005, A\&A, 438, 1149) that proposes a theoretical development for their atmospheric and oceanic excitation.
\end{abstract}

Key words. reference systems - Earth

\section{Introduction}

VLBI is currently the only method available for measuring the sidereal orientation of the Earth. The parameters involved are universal time, precession and nutation.

The Precession-Nutation model recommended in 2000 by the International Astronomical Union, IAU 2000, was derived by Mathews et al. (2002). These authors used estimates of the largest nutation coefficients to determine a set of physical parameters that influence the response of the real Earth to external torques, in the context of a model of the Earth's interior. In this adjustment, all VLBI observations available over 1980.02002.5 were used.

The estimation of the amplitude of precession and nutation terms consists of a least-squares extraction of periodic component at known frequencies from the motion of the Earth's axis of figure relative to a conventional celestial reference frame that is materialised by coordinates of a set of extragalactic radio sources. A fundamental hypothesis in this method is that the directions of the fiducial objects stay fixed in time. The current definition of the International Celestial Reference Frame ICRF (Ma et al. 1998) is based on this hypothesis. If, due to the intense activity of the celestial objects taken as reference points, some of them have position instabilities or apparent motion, the celestial reference frame will exhibit local inhomogeneities that may vary in time. As a result, some nutation coefficients derived may be contaminated. Dehant et al. (2003) showed that inhomogeneity in the celestial frame can lead to changes of nutation coefficients estimates at the level of a few tens of microarcseconds ( $\mu$ as).

Although the causes that induce variations of the sidereal rotation of the Earth at all periods from minutes to millennia are globally known, there is no predictive model comparable to those available for precession and the forced nutations, that leave only about $10^{-9}$ of the phenomenon unexplained, to be observed and further explained. Fine tests on the influence of the celestial reference frame on periodic variations in universal time determinations are therefore impossible. The tests have to be restricted to simple models. 
In a recent study of individual source motions, Feissel-Vernier (2003) devised a stability selection scheme that pinpointed 163 well observed sources that are highly unstable or even drifting in the 1990-2002 time frame. Conversely, a set of 199 stable well observed sources was identified. The use of the stable sources in the definition of the axes of a celestial reference frame was shown to lower the medium-term instability of the celestial reference frame from 28 to $6 \mu$ as when compared to the ICRF. Another set of 358 sources were observed too sparsely for a statistical evaluation of their stability.

Arias \& Bouquillon (2004) showed that this core of stable sources allows a better match of celestial reference frames attached to the ICRF by various authors. Gontier \& Feissel-Vernier (2003) showed that the derived time series of pole coordinates and universal time were more stable when excluding the unstable sources than those relative to all sources, as currently done in operational programs.

In this paper, we study the impact that the consideration of the stability of reference extragalactic radio sources may have on the precise knowledge of the sidereal orientation of the Earth, namely free or forced nutations of the Earth, precession, obliquity rate and universal time.

We first recall in Sect. 2 the main analysis strategies used in the construction of celestial reference frames from VLBI observations. In Sect. 3 we study the random deformations of celestial reference frames associated with various source analysis strategies. The size of these deformations may give an idea of the improvement expected from the rejection of unstable or drifting sources.

We develop in Sect. 4 the model for using time series of radio source coordinates to infer the robustness of observations of precession and nutation coefficients, and of the low frequency components in universal time.

In Sect. 5 through 7 we describe the impact of the reference sources selection on the determination of the secular terms, the 18.6-year component, and of some medium term nutation components that are believed to be influenced by the atmosphere or the oceans, namely the annual nutation, the Free Core Nutation and the Free Inner Core Nutation. The impact of the reference sources selection on UT1 is evaluated in Sect. 8.

\section{VLBI celestial reference frames}

The celestial reference frames that are implicit in the determination of precession and nutation are usually based on global VLBI observations since 1980. Table 1 gives the yearly observations rates used for the celestial reference frame since 1984, in terms of numbers of sources observed and numbers of sessions. Figure 1 shows the distribution history of these observations as a function of the source declinations. The 1980-83 data, sparser and less precise, are not shown. Observations with the southern hemisphere stations started only in 1989 and became less frequent after 1996. New sources were progressively added in the northern hemisphere, in particular in 1990 and in 1995. Only some of them were still observed after 2000-2001. The number of yearly sessions had a plateau above 200 per year over 1990-1994. Since then, it stabilised around 130 per year.
Table 1. VLBI yearly rates of observations available for the celestial reference frame in terms of number of sources (srces) and sessions (sess).

\begin{tabular}{crrccc}
\hline \hline Central & \multicolumn{2}{c}{ Number of } & \multicolumn{2}{c}{ Central } & \multicolumn{2}{c}{ Number of } \\
date & srces & sess & date & srces & sess \\
\hline 1984.0 & 51 & 93 & 1993.0 & 312 & 219 \\
1985.0 & 57 & 119 & 1994.0 & 311 & 203 \\
1986.0 & 62 & 134 & 1995.0 & 359 & 170 \\
1987.0 & 119 & 154 & 1996.0 & 307 & 132 \\
1988.0 & 183 & 186 & 1997.0 & 321 & 142 \\
1989.0 & 220 & 197 & 1998.0 & 321 & 129 \\
1990.0 & 234 & 255 & 1999.0 & 360 & 133 \\
1991.0 & 288 & 236 & 2000.0 & 301 & 116 \\
1992.0 & 325 & 251 & 2001.0 & 276 & 127 \\
\hline
\end{tabular}

The analysis strategies for deriving a celestial reference frame from multi-year VLBI observations include a number of choices. Two of them are considered here.

- A first choice has to be made concerning the definition of the celestial and terrestrial reference frames and their connection in time. Two different approaches are used, as follows.

- In the derivation of the ICRF and its extensions (Ma et al. 1998; Fey et al. 2004), the so-called CRF approach was used, i.e. station positions are set as arc parameters, thereby estimated independently for each observing session. Polar motion and universal time are not estimated but nutation corrections (celestial pole offsets) are obtained. This strategy was chosen in order to free the celestial frame solution from systematic errors that may be propagated from terrestrial network deficiencies.

- In this study, we consider solutions based on the socalled TRF approach, in which most station positions and velocities are set as global parameters, i.e. considered constant over the total data span. Polar motion, universal time, and nutation corrections are estimated for each session. Comparisons performed on test solutions with data up to 2002.7 (Feissel-Vernier et al. 2004) have shown that keeping the link between the celestial and the terrestrial reference frames does not impact the quality of the precession-nutation measurements. This might not have been the case when Ma et al. (1998) derived the original ICRF based on observations before 1995.

- Some other features of the analyses are worth mentioning in the context of this study:

- The observed sources may be treated either as global sources, when their equatorial coordinates $(\alpha, \delta)$ are considered fixed over the total observation span and estimated as two constants, or as arc sources, when their coordinates are estimated independently for each session in which the source is observed. In the case of simultaneous estimation of nutation angles, only the global sources contribute to this estimation. 


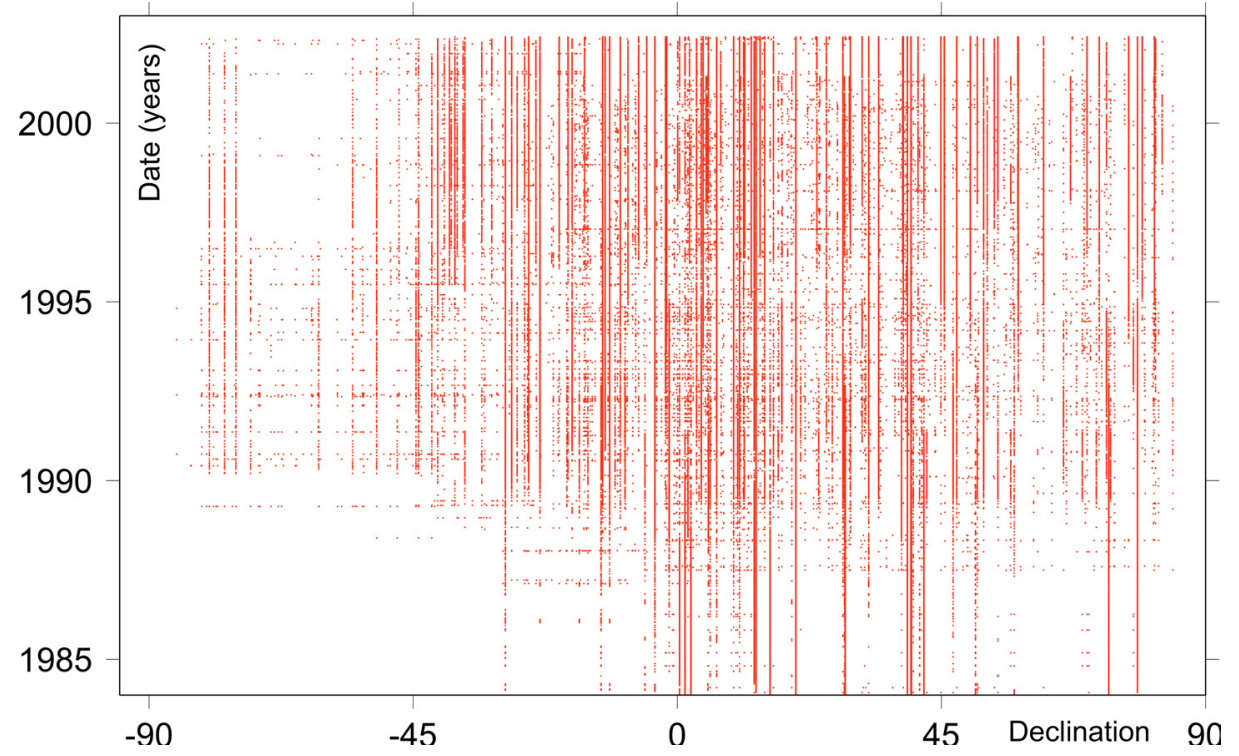

Fig. 1. VLBI observations history, 1984-2002. Each dot represents a multibaseline VLBI session where the source with a given declination was observed.

- In order to attach the axes of the derived celestial reference frame to the reference ICRF axes, a no-netrotation (NNR) condition is implemented, using a set of selected global sources, e.g. the defining sources in the case of the ICRF, or the stable sources in this study.

To study the influence of the effective celestial reference frame on determination of precession and nutation coefficients, we consider two solutions for the celestial reference frame and the celestial pole offsets based on the 1980.0-2002.7 data, described in Table 2. Data was analyzed with the CALC-SOLVE software package.

- In the first solution, referred to as Conventional, all sources are considered global and the NNR condition with respect to the ICRF is based on the 212 ICRF defining sources. Precession and nutation studies based on VLBI observations usually follow that scheme.

- A second solution, referred to as Proposed, is studied. In this solution, the 163 unstable sources (according to Feissel-Vernier 2003) are treated as arc sources, and all other sources, i.e. 199 stable sources and 358 sparsely observed sources, are treated as global. The NNR condition with respect to the ICRF is only based on the 199 stable sources. This solution, where the derived celestial pole offsets $(\mathrm{d} \psi, \mathrm{d} \epsilon)$ are made to refer to these selected sources, will be used to infer the impact of source selection on precession and nutation determinations. This solution keeps as global the sparsely observed sources, which represent less than $10 \%$ of the total observational data. One reason for considering these sources as global is that it leads to a cleaner wavelet spectrum (see Sect. 7.2) than when restricting the set of global sources to the stable ones, which may be due to better source distribution in the individual sessions from which celestial pole offsets are estimated.
Table 2. Two VLBI celestial reference frames under study.

\begin{tabular}{cccc}
\hline \hline $\begin{array}{c}\text { Name } \\
\text { of frame }\end{array}$ & $\begin{array}{c}\text { Global } \\
\text { sources }\end{array}$ & $\begin{array}{c}\text { Arc } \\
\text { sources }\end{array}$ & $\begin{array}{c}\text { NNR } \\
\text { sources }\end{array}$ \\
\hline Conventional & All & none & ICRF def. \\
& $(724)$ & & $(212)$ \\
Proposed & Sparse & Unstable & Stable \\
& $(363)$ & $(163)$ & $(199)$ \\
& Stable & & \\
\hline
\end{tabular}

\section{Internal consistency of celestial reference frames}

Two celestial reference frames can be compared by evaluating their relative rotation angles $A_{1}, A_{2}, A_{3}$ around the axes of the equatorial coordinate system, using the following equations, where $\alpha, \delta$ are the source coordinates and $\Delta \alpha, \Delta \delta$ are the differences of coordinates in the two frames.

$\Delta \alpha=A_{1} \tan \delta \cos \alpha+A_{2} \tan \delta \sin \alpha-A_{3}$

$\Delta \delta=-A_{1} \sin \alpha+A_{2} \cos \alpha+\mathrm{d} z$

The $\mathrm{d} z$ parameter absorbs systematic differences in declination that may be caused by inaccuracy of the tropospheric propagation correction for sources observed at low elevations, which is often the case for sources in the equatorial region as a result of station network geometry. In the pre-ICRF celestial frames, significant values of $\mathrm{d} z$ were found when comparing solutions obtained by different analysts. However, McMillan \& Ma (1997) showed that adding the so-called gradient parameter when modelling the tropospheric delay minimises this defect. The gradient parameterisation is now commonly used in VLBI analysis.

To evaluate the internal consistency of the two celestial reference frames of Table 2, we compare them with 
Table 3. Standard deviations of source positions in the two celestial reference frames under study.

\begin{tabular}{llc}
\hline \hline Reference & \multicolumn{2}{c}{ Standard deviations } \\
frame & $\Delta \alpha \cos \delta$ & $\Delta \delta$ \\
\hline Proposed & $27.1 \mu$ as & $21.2 \mu$ as \\
Conventional & $28.3 \mu$ as & $24.0 \mu$ as \\
\hline
\end{tabular}

two independent celestial frames available at the IERS/ICRS Product Center (ICRS-PC, 2003), one provided by the IAA (St Petersburg) and the other by the BKGI (Leipzig). Note that the Proposed frame of Table 2 was rotated to the ICRS axes using an NNR condition based on the 199 stable sources, while for the other three reference frames the NNR condition was based on the 212 ICRF defining sources.

In each comparison, the two frames are rotated to common axes in a least-squares adjustment of the above equations applied to the 206 sources that are common to the four reference frames. About $2 / 3$ of them belong to the stable category, with the other $1 / 3$ sparsely observed. Variances of the postfit residuals $\Delta \alpha \cos \delta$ and $\Delta \delta$ are computed. In this process, the estimated parameters $A_{1}, A_{2}, A_{3}$, and $\mathrm{d} z$ absorb systematic differences that can be expected from the construction schemes or the compared reference frames. In effect, the estimated parameters may reach $50 \mu$ as for the rotation angles and $70 \mu$ as for $\mathrm{d} z$. The variance of the residuals measures only the random differences.

The three-cornered-hat method is then applied to these variances. This method allows us to estimate the variance of the noise of three or more sets of measurements of the same quantities, here the source right ascensions and declinations, under the assumption that the errors are independent in the three data sets. Considering the variances of the differences between two data sets $i$ and $j$, one can write

$$
\begin{aligned}
& \operatorname{var}\left(\alpha_{i} \cos \alpha-\alpha_{j} \cos \alpha\right)=\operatorname{var}\left(\alpha_{i} \cos \alpha\right)+\operatorname{var}\left(\alpha_{j} \cos \alpha\right) \\
& \operatorname{var}\left(\delta_{i}-\delta_{j}\right)=\operatorname{var}\left(\delta_{i}\right)+\operatorname{var}\left(\delta_{j}\right)
\end{aligned}
$$

If three reference frames are considered, one gets three sets of the above equations that can be solved to obtain the individual noise variances $\operatorname{var}\left(\alpha_{k} \cos \alpha\right)$ and $\operatorname{var}\left(\delta_{k}\right)$ for $k=1,3$. If more than three data sets with independent errors are available, the individual noise variances can be estimated by means of a statistical algorithm, e.g. by least squares.

Table 3 gives the individual standard deviations thus derived for the two reference frames described in Table 2. Direct comparison of the two frames of Table 2 was indeed not used in this process, as their errors cannot be assumed to be independent. Standard deviations of the external frames (not shown) are in the same range of values. The robustness of the estimations was tested by associating the data in various ways, and the results remain stable within $2 \mu$ as.

Table 3 shows that orienting the frame using the stable sources Proposed reference frame improves the variance of residuals by $9 \%$ in right ascension and $28 \%$ in declination, when compared to conventional practice. One can expect that improvement in the internal consistency corresponds to better time stability of the frame, which in turn leads to better determined precession and nutation coefficients.

\section{Perturbation of precession and nutation determinations by radio source instability}

To what extent can the time dependent deformations of the reference frames contaminate the estimation of precession or nutation coefficients? To estimate the magnitude of this possible effect, we evaluate the celestial pole apparent motion resulting from the sources' motions and we look for possible signatures at frequencies in the nutation spectrum, or linear drifts, that would be interpreted as systematic errors on the corresponding precession-nutation components. If the pole motion is described by the rotation angles $A_{1}(t), A_{2}(t)$ (see Sect. 3), the induced variations of $\Delta \psi(t)$ and $\Delta \epsilon(t)$ are:

$A_{1}(t)=\Delta \epsilon(t)$

$A_{2}(t)=-\Delta \psi(t) \sin \epsilon_{0}$.

Combining these relationships with (1) and (2), we obtain a model for estimating spurious nutation or precession terms induced by the neglect of source apparent motion. The observation of $\Delta \alpha(t)$ and $\Delta \delta(t)$ at epoch $t$ of a radio source with coordinates $\alpha$ and $\delta$ provides two observation equations of the form:

$\Delta \alpha(t)=\tan \delta \cos \alpha\left\{\epsilon_{\mathrm{J} 2000}+\dot{\epsilon}(t-\mathrm{J} 2000)\right.$

$\left.+\sum_{i}\left[E_{i} \sin 2 \pi \omega_{i}(t-\mathrm{J} 2000)+F_{i} \cos 2 \pi \omega_{i}(t-\mathrm{J} 2000)\right]\right\}$

$-\tan \delta \sin \alpha \sin \epsilon_{0}\left\{\psi_{\mathrm{J} 2000}+\dot{\psi}(t-\mathrm{J} 2000)\right.$

$\left.+\sum_{i}\left[P_{i} \sin 2 \pi \omega_{i}(t-\mathrm{J} 2000)+Q_{i} \cos 2 \pi \omega_{i}(t-\mathrm{J} 2000)\right]\right\}$

$-A_{3}-\dot{A_{3}}(t-\mathrm{J} 2000)$

$\Delta \delta(t)=-\sin \alpha\left\{\epsilon_{\mathrm{J} 2000}+\dot{\epsilon}(t-\mathrm{J} 2000)\right.$

$\left.+\sum_{i}\left[E_{i} \sin 2 \pi \omega_{i}(t-\mathrm{J} 2000)+F_{i} \cos 2 \pi \omega_{i}(t-\mathrm{J} 2000)\right]\right\}$

$-\cos \alpha \sin \epsilon_{0}\left\{\psi_{\mathrm{J} 2000}+\dot{\psi}(t-\mathrm{J} 2000)\right.$

$\left.+\sum_{i}\left[P_{i} \sin 2 \pi \omega_{i}(t-\mathrm{J} 2000)+Q_{i} \cos 2 \pi \omega_{i}(t-\mathrm{J} 2000)\right]\right\}$

$+\mathrm{d} z$.

The unknown parameters $\dot{\psi}$ and $\dot{\epsilon}$ would give the effect on the estimated precession constant and obliquity rate, respectively. $P_{i}$ and $Q_{i}$ are the in phase and out of phase components of a nutation in longitude with frequency $\omega_{i}$, be it forced or free. $E_{i}$ and $F_{i}$ are the corresponding components in obliquity. The unknown parameter $\mathrm{d} z$ of Eq. (8) is considered fixed over the time span considered, and its value is found to be consistent with 0 within $\pm 10 \mu$ as over 1984-2002.

The behaviour of the parameters $A_{3}$ and $\dot{A_{3}}$ in Eq. (7) gives information about the stability of the origin of right ascensions of the celestial reference frames corresponding to the various 


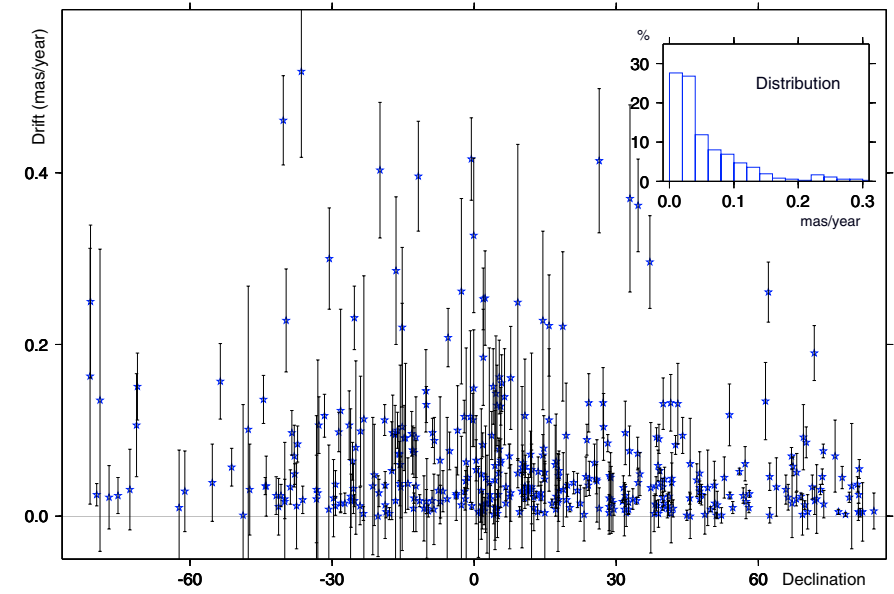

Fig. 2. Sources linear drifts over 1989.5-2002.4 in the direction of their maximum variance, as a function of declination. The insert shows the histogram of the observed drifts.

source selections considered. Changes in universal time are connected to changes in $A_{3}$ and the corresponding terrestrial frame angle $R_{3}$ by the following relationship:

$\mathrm{d} U T 1=\mathrm{d} A_{3}-\mathrm{d} R_{3}$.

The impact of source instability on the celestial orientation of the Earth's axis is evaluated using relationships (7) and (8) in Sect. 5 for precession, Sect. 6 for the 18.6-year nutation component, Sect. 7 for the annual component and the core and inner core free nutations, and in Sect. 8 for universal time, using relationships (7) and (9).

The source apparent motions are taken from the data used by Feissel-Vernier (2003). Perturbation evaluated in this way can only be considered as an approximate correction to analysis of the time series of $\Delta \psi, \Delta \epsilon$, and $U T 1$, as the latter are obtained from each session with a different subset of the considered celestial frame. Nevertheless, they are useful as a rough estimate of the size of perturbation.

\section{Precession and obliquity rate}

The apparent motion of a number of the observed radio-source apparent motions may be modelled as linear velocities in some preferred direction, probably connected to the directions of the jet. Figure 2 shows least-square estimates of source linear drifts over 1989.5-2002.4 as a function of declination. The inserted histogram shows that about $1 / 3$ of the sources have apparent velocities larger than $60 \mu \mathrm{as} / \mathrm{year}$. Although the distribution of their directions may be expected to be random, it is necessary to check at what level they can mimic linear drifts in longitude or in obliquity.

As explained in Sect. 4, we estimate precession and obliquity rate corrections as the linear terms obtained by a leastsquares fit of individual source coordinates to Eqs. (7) and (8) for a given set of radio source coordinates. Table 4 gives the values of the precession correction and of the obliquity rate that refers either to the Proposed celestial reference frame or to the Conventional one, along with the estimated perturbation of linear trends due to source instability. The latter
Table 4. Precession and obliquity rate corrections: role of the source selection.

\begin{tabular}{|c|c|c|c|}
\hline $\begin{array}{c}\text { Source } \\
\text { selection }\end{array}$ & $\begin{array}{l}\text { Precession } \\
\mu \text { as/year }\end{array}$ & $\begin{array}{c}\text { Obliqu. rate } \\
\mu \text { as/year }\end{array}$ & $\begin{array}{c}\text { StDev } \\
(\mu \text { as })\end{array}$ \\
\hline & \multicolumn{2}{|c|}{ a) VLBI-IAU 2000} & $/$ sess \\
\hline Proposed & $-55.9 \pm 4.9$ & $-30.9 \pm 1.8$ & 235 \\
\hline \multirow[t]{2}{*}{ Conventional } & $-18.0 \pm 4.3$ & $-29.5 \pm 1.7$ & 243 \\
\hline & \multicolumn{2}{|c|}{ b) Correction for source instability } & /srce \\
\hline Proposed & $7.2 \pm 1.7$ & $2.9 \pm 0.3$ & 246 \\
\hline Conventional & $5.9 \pm 1.2$ & $4.3 \pm 0.2$ & 256 \\
\hline Unstable only & $6.6 \pm 1.7$ & $7.9 \pm 0.5$ & 275 \\
\hline \multicolumn{4}{|c|}{ c) VLBI-IAU 2000 corrected } \\
\hline Proposed & $-48.7 \pm 5.2$ & $-28.0 \pm 1.8$ & \\
\hline Conventional & $-12.1 \pm 4.5$ & $-25.2 \pm 1.7$ & \\
\hline
\end{tabular}

component should be added to results derived from the series of $\Delta \psi$ and $\Delta \epsilon$ to tentatively free them from the source motion effect. In both cases the linear trends are estimated over the time span 1984.0-2002.7 (about 3120 24-h sessions) simultaneously with an 18.6-year term (see results in Table 5).

The values of VLBI-IAU 2000 corrected for the estimated effect of source instabilities are listed at the bottom of Table 4 with uncertainties that result from the quadratic addition of the two estimates. The obliquity rate correction is not negligible but is only slightly affected by apparent source motions. The precession correction is more affected by the source selection, especially considering the Proposed reference frame. Correction for apparent source motion effect tends to reconcile the two estimates but does not explain all of the difference. Note that perturbation induced by the 163 unstable sources alone is not particularly large.

Gontier et al. (2001) have shown that the general improvements in VLBI technology and observing strategies brought the astrometric results to their current precision towards the end of the 1980's. Starting about 1990, individual source coordinates stabilised. While the early data still play an important role in the study of precession, the possibility that their relatively high noise creates systematic errors in the estimated pole rate cannot be ruled out. To check this possible effect, test computations of linear trends considering time periods before and after 1990 were performed, using a common correction for the 18.6-year nutation term. They show stability in the precession corrections that is consistent with the uncertainties in Table 4. The instability of the obliquity rate is larger: up to $6 \mu$ as/year, i.e. about three times the Table 4 uncertainties.

Table 4 also gives the standard deviations of post-fit residuals for $\Delta \psi \sin \epsilon_{0}$ and $\Delta \epsilon$ per session in analysis of VLBIIAU 2000 in the first section, and for $\Delta \alpha \cos \delta$ and $\Delta \delta$ per source and per session in analysis of the source apparent motions in the second section. While the first category measures the level of unresolved noise for a complete session that includes several hundred observations in a 24-h time span, the second one corresponds to the complete set of sources observed over the 19-year time span. 
Table 5. Estimation of the 18.6-year nutation term: role of the source selection. Unit: $\mu$ as.

\begin{tabular}{lrccc}
\hline \hline \multicolumn{1}{c}{$\begin{array}{c}\text { Source } \\
\text { selection }\end{array}$} & sine & cosine & sine & cosine \\
\hline \multicolumn{5}{c}{ a) VLBI-IAU 2000 } \\
Proposed & $251 \pm 23$ & $154 \pm 26$ & $-13 \pm 9$ & $23 \pm 10$ \\
Conventional & $80 \pm 20$ & $163 \pm 24$ & $-45 \pm 8$ & $26 \pm 10$ \\
& \multicolumn{5}{c}{ b) Correction for source instability } \\
Proposed & $19 \pm 93$ & $14 \pm 47$ & $-36 \pm 8$ & $-12 \pm 2$ \\
Conventional & $146 \pm 81$ & $58 \pm 39$ & $-21 \pm 6$ & $-7 \pm 2$ \\
Unstable only & $556 \pm 161$ & $178 \pm 74$ & $0 \pm 9$ & $8 \pm 3$ \\
& \multicolumn{5}{c}{ c) VLBI-IAU 2000 corrected } \\
Proposed & $270 \pm 95$ & $168 \pm 53$ & $-49 \pm 12$ & $12 \pm 10$ \\
Conventional & $226 \pm 83$ & $221 \pm 46$ & $-66 \pm 10$ & $19 \pm 10$ \\
\hline
\end{tabular}

\section{The 18.6-year nutation}

Table 5 provides similar quantities to Table 4 for the 18.6-year term. Corrections in obliquity $(\Delta \epsilon)$ for the two source selections are close to each other, as are the estimated source instability corrections. The results are moderately affected by the source selection. The case of longitude $(\Delta \psi)$, where the largest discrepancy between observations and model is mentioned by Mathews et al. (2002), is quite different. Amplitudes of the in-phase components differ by about $170 \mu$ as depending on the source selection. The estimated source instability correction lessens the difference but does not fully reconcile the estimates. Note that perturbation by the 163 unstable sources alone is large and noisy. Nevertheless, both estimates confirm a discrepancy in the observations sing the IAU 2000 model with a total amplitude of about $320 \mu$ as. The sizeable level of the out of phase part suggests that the cause of these remaining discrepancies may be found in variable geophysical phenomena not considered in the model.

\section{Time varying nutation components in the seasonal and interseasonal frequency band}

In this section, we investigate the impact of source selection on the determination of variable nutation terms, namely:

- the Free Core Nutation (FCN) with a 431-day period, a free mode which may or may not be excited, e.g. by the atmosphere, depending on the time span considered;

- the retrograde component of the annual term, which includes a part that is forced by the atmospheric pressure diurnal variations, with an observable response due to the proximity of Free Core Nutation;

- the Free Inner Core Nutation (FICN), a prograde free oscillation with an assumed period of 1025 days in the Mathews et al. (2002) model and a suggested period of 880 days according to Dehant et al. (2003).
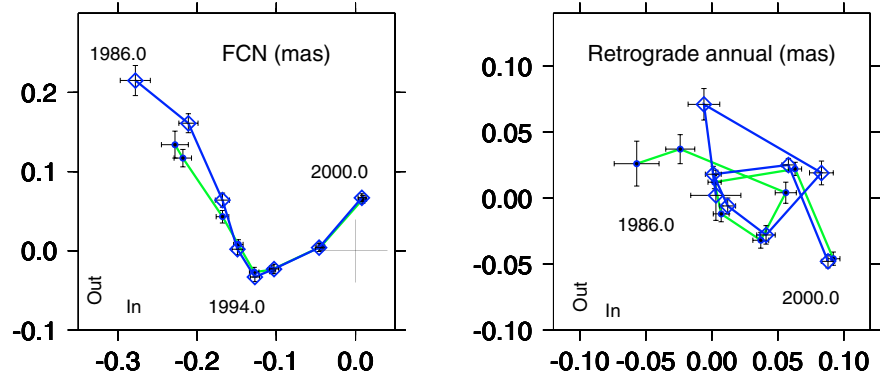

Fig. 3. Time variation in amplitude and phase of the Free Core Nutation and of the retrograde annual nutation, derived from VLBI observations of either the Proposed reference frame (blue/dark) or the Conventional one (green/light).

\subsection{Retrograde FCN and annual component}

Investigating variations in the annual and FCN terms requires considering six-year data spans in order to decorrelate the two components. Figure 3 shows the time variation of the retrograde part of these two components. These variations are shown for both cases of the Proposed and the Conventional celestial reference frames. Time evolution of the FCN shows a remarkable change with time of both the phase and amplitude, with a $200 \mu$ as amplitude in the 1980 s and about $50 \mu$ as at the end of the 1990s. The variation is more pronounced in the early years when considering the selected sources. According to Dehant et al. (2003), the enhanced FCN in the 1980s was excited by the atmosphere. The time evolution of the retrograde annual term stays within a diameter of $100 \mu \mathrm{as}$, as expected from the knowledge of atmospheric diurnal excitation. The source selection only influences the early data.

\subsection{Prograde FICN}

The prograde spectral residual content of the series of VLBIderived celestial pole offsets corrected for the IAU 2000 Nutation model is described in Figs. 4 and 5 in the form of wavelet analysis (Dehant et al. 2003). Figure 4, which considers all sources (Conventional approach), shows energy in the 1984-1990 time frame that disappears after 1990, in agreement with the Gontier et al. (2001) stability diagnosis mentioned in Sect. 5. Moreover, according to Dehant et al. (2005), the level of energy required from the atmospheric and oceanic excitation exceeds by several orders of magnitude the actual levels. In Fig. 5 that corresponds to the Proposed approach the pre-1990 highs disappear. However, thanks to a largely enhanced sensitivity of the scalogramme, a weaker peak with a 800-900 days period appears over 1990-1995. The presence of this peak led to the two following investigations

- the energy available in the diurnal band of the atmosphere and ocean, which would excite the FICN free mode (Dehant et al. 2005); and

- the level of confidence of numerical detection of this term, considering the source selection and propagation of their instability.

The search for FICN provides an opportunity to test the detectability of weak variable oscillations by VLBI in the 


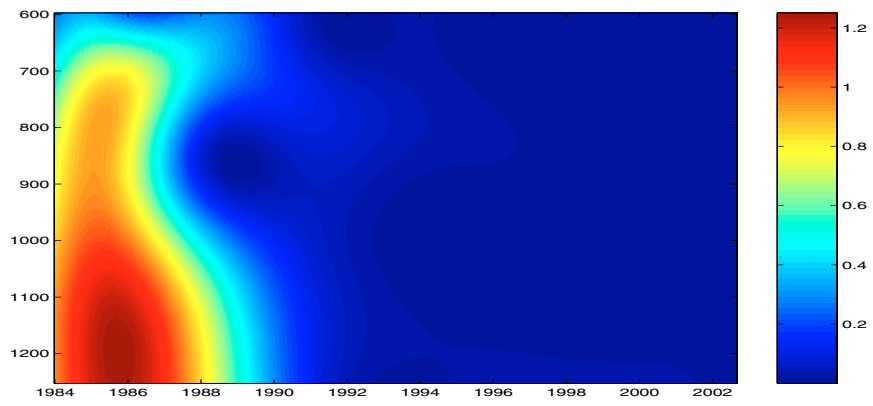

Fig. 4. Conventional celestial reference frame: wavelet analysis of the prograde nutation over 1984-2002. The data are residuals to the IAU 2000 Nutation model. The vertical variable is the period in days and the horizontal variable the date in years. The colour scale is shown on the side in arbitrary units.

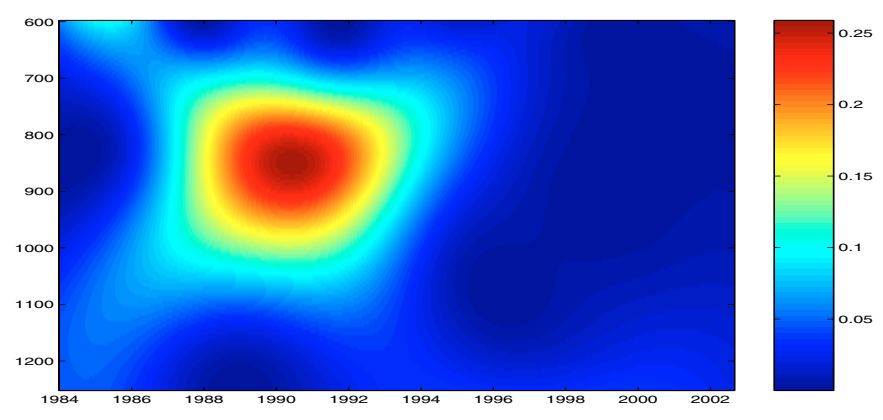

Fig. 5. Same as Fig. 4 for the Proposed celestial reference frame. Note the dramatic change in the colour scale.

interannual frequency band. Assuming a period of 880 days, we analyse the VLBI series of $\Delta \psi$ and $\Delta \epsilon$, together with a 431-day term over three successive five-year data spans (Table 6). This 1800-day length of time contains twice the period of interest, while it is a good compromise to accommodate the variable FCN shown in Fig. 3. The VLBI estimate (upper part of Table 6) exhibits a potential candidate for detecting the FICN in 1990 through 1994 with a prograde amplitude $37 \pm 10 \mu$ as when considering the Proposed reference frame and $24 \pm 10 \mu$ as when considering all sources. However, if we consider the correction for source instability in both cases, the amplitudes are deflated, in particular in the case of the Proposed reference frame. We therefore conclude that the prograde oscillation that appears in various spectra and least-squares estimations is most probably an artefact due to radio source instability.

\section{Universal time and the stability of the right ascension origin}

Possible contamination of the stability of the origin of right ascensions by the source instability is modelled as the orientation at J2000.0 and a linear variation. Values estimated over 1989.5-2002.4 are listed in Table 7. The direction of the origin is affected at the level of $\pm 10 \mu$ as, corresponding to less than one microsecond in universal time. The linear drift estimate in $A_{3}$ is more scattered than those of the precession and obliquity rate corrections, which suggests some systematism in the apparent motions in declination. The spurious linear drift for the conventional and proposed source selections stays
Table 6. Estimated in phase and out of phase amplitudes of a potential prograde FICN with a 880-day period. The origin of phases is 2000.0. Unit: $\mu$ as.

\begin{tabular}{ccrrr}
\hline \hline Time & \multicolumn{2}{c}{ Proposed } & \multicolumn{2}{c}{ Conventional } \\
span & In & Out & In & Out \\
\hline \multicolumn{5}{c}{ a) VLBI-IAU 2000 } \\
$1985-1989$ & $0 \pm 15$ & $-21 \pm 15$ & $11 \pm 14$ & $15 \pm 14$ \\
$1990-1994$ & $\mathbf{1 7} \pm \mathbf{7}$ & $\mathbf{- 3 3} \pm \mathbf{7}$ & $\mathbf{1 6} \pm \mathbf{7}$ & $\mathbf{- 1 8} \pm \mathbf{7}$ \\
$1995-1999$ & $6 \pm 6$ & $3 \pm 6$ & $13 \pm 6$ & $4 \pm 6$ \\
\multicolumn{5}{c}{ b) Correction for source instability } \\
$1985-1989$ & $-22 \pm 92$ & $4 \pm 59$ & $6 \pm 77$ & $22 \pm 46$ \\
$1990-1994$ & $\mathbf{- 5} \pm \mathbf{4 4}$ & $\mathbf{2 4} \pm \mathbf{1 9}$ & $\mathbf{- 4} \pm \mathbf{3 5}$ & $\mathbf{2 2} \pm \mathbf{1 5}$ \\
$1995-1999$ & $3 \pm 29$ & $2 \pm 14$ & $3 \pm 25$ & $5 \pm 12$ \\
\multicolumn{5}{c}{ c) VLBI-IAU 2000 corrected } \\
1985-1989 & $-22 \pm 93$ & $-17 \pm 61$ & $17 \pm 78$ & $37 \pm 48$ \\
$1990-1994$ & $\mathbf{1 2} \pm \mathbf{4 5}$ & $\mathbf{- 9} \pm \mathbf{2 0}$ & $\mathbf{1 2} \pm \mathbf{3 6}$ & $\mathbf{4} \pm \mathbf{1 7}$ \\
$1995-1999$ & $9 \pm 30$ & $5 \pm 15$ & $16 \pm 26$ & $9 \pm 13$ \\
\hline \multicolumn{5}{c}{}
\end{tabular}

Table 7. Corruption of the origin of right ascensions by the radio source instability estimated over the time span 1989.5-2004.4.

\begin{tabular}{|c|c|c|}
\hline $\begin{array}{c}\text { Source } \\
\text { selection }\end{array}$ & $\begin{array}{c}A_{3} \\
\mu \text { as }\end{array}$ & $\begin{array}{c}\mathrm{d} A_{3} / \mathrm{d} t \\
(\mu \text { as/year) }\end{array}$ \\
\hline Proposed & $-7.5 \pm 12.4$ & $-3.1 \pm 2.9$ \\
\hline Conventional & $1.5 \pm 10.6$ & $0.5 \pm 2.3$ \\
\hline Unstable only & $26.5 \pm 20.0$ & $7.8 \pm 4.0$ \\
\hline
\end{tabular}

under $6 \mu$ as/year, corresponding to about 0.5 microsecond/year in universal time. Note the slightly larger values for the proposed selection source when compared to the conventional one. This corresponds to the appearance of a peak in the spectrum of the right ascension residuals with a period of six years and an amplitude of $15 \mu$ as, corresponding to one microsecond in universal time.

\section{Conclusion}

We showed that taking the most stable sources as the basis for defining the celestial reference frame orientation and for treating the most unstable sources as arc sources improves the internal consistency of the reference frame at the level of $30 \%$ in the variance of source declinations. As a result, determination of precession and nutation components is affected at various levels, depending on their frequency, as summarized hereafter.

- In the seasonal and FCN frequency band, the effect is small in the 1980s and negligible in the 1990s. In the two- to three-year frequency band, we showed that, even when considering the most stable sources, source instabilities may mimic transient oscillations with an amplitude up to $20 \mu$ as.

- For what concerns the principal term of nutation, actual departure of VLBI results with the IAU 2000 model may reach $50 \mu$ as in $\Delta \epsilon$ and $320 \mu$ as in $\Delta \psi$. The latter discrepancy is larger than the $80 \mu$ as discrepancy mentioned by Mathews et al. (2002).

- The influence on the determination of the precession correction and the secular obliquity rate is lower than $10 \mu$ as. 
The precession correction departure from the IAU 2000 model, excluding the unstable sources, is somewhat larger than the one based on all sources. This discrepancy is not modified by the source instability effect. Finally, departures from the IAU 2000 values stay around $50 \mu$ as/year in precession and $30 \mu \mathrm{as} /$ year in obliquity rate.

Because there is converging evidence that considering source stability improvemes the internal consistency of VLBI-derived celestial reference frames brought by the consideration of source stability, we suggest that future investigations of precession and nutation of the global Earth take full account of this new aspect of the VLBI data.

The contribution of instability of right ascensions to the instability of VLBI-derived UT1 was found to stay under one microsecond at J2000.0 and 0.5 microsecond/year for both the conventional and proposed source selections. Analysis of results based on the proposed source selection suggests a common effect in right ascension, with a period of six years and an amplitude of $15 \mu$ as.

\section{References}

Arias, E. F., \& Bouquillon, S. 2004, 422, 1105

Dehant, V., Feissel-Vernier, M., de Viron, O., et al. 2003, JGRB, 108, 2275

Dehant, V., de Viron, O., \& Greff, M. 2005, A\&A, 438, 1149

Dehant, V., de Viron, O., Feissel-Vernier, M., \& Rivoldini, A. 2003, AGU Fall Meeting

Feissel-Vernier, M. 2003, A\&A, 403, 105

Feissel-Vernier, M., Ma, C., \& Gontier, A.-M. 2004, IERS TN in preparation, ed. C. Ma

Fey, A. L., Ma, C., Arias, E. F., et al. 2004, AJ, 127, 3587

Gontier, A.-M., Le Bail, K., \& Feissel, M. 2001, A\&A, 375, 661

Gontier, A.-M., \& Feissel-Vernier, M. 2003, IAU XXV, Proc. JD16 (Gaume, McCarthy, Souchay eds)

ICRS Product Center 2003, http://hpiers.obspm. fr/icrs-pc/, click Compared CRF

Ma, C., Arias, E. F., Eubanks, T. M., et al. 1998, AJ, 116, 516

Mathews, P. M., Herring, T. A., \& Buffett, B. A. 2002, JGRB, 107, 1029

McMillan, D. S., \& Ma, C. 1997, GRL, 24, 453 